transmitted infections (STI). Providers struggled to differentiate event-specific anxieties from more chronic, underlying anxietyrelated conditions. Three barriers constrained the providers' abilities to effectively address MHSU service needs: 1) clinic mandates or funding models (specific to STI/HIV or reproductive health); 2) 'silo-ing' (i.e., physical and administrative separation) of services; and, 3) limited familiarity with MSHU service referral pathways. In response to these barriers and acknowledging the prevalence and prominence of MHSU concerns among clients, participants described actionable solutions. 1) Reduce silos, by clarifying referral pathways from sexual health clinics to MHSU providers. 2) Co-locate sexual health and MHSU services. 3) Assess the broader health needs of high-anxiety and low-STI risk clients who frequently access sexual health services.

Conclusion Sexual health clinicians in British Columbia generally affirm the results of previous, quantitative and clientfocused research showing high rates of MHSU-related needs among sexual health clinic clients. Providers prioritized specific short-term (referral-focused) and long-term (healthcare reorganization) solutions for improving access to MHSU for those using sexual health services.

Disclosure No significant relationships.

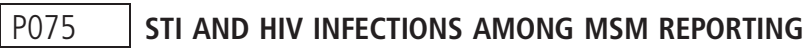 EXPOSURE TO GONORRHEA OR CHLAMYDIA: IMPLICATIONS FOR EXPEDITED PARTNER THERAPY}

${ }^{1}$ Julia Schillinger*, ${ }^{2}$ Kelly Jamison, ${ }^{2}$ Jennifer Slutsker, ' Susan Blank. ${ }^{1}$ Centers for Disease Control and Prevention, Division of STD Prevention, New York City, USA; ${ }^{2}$ New York City Department of Health and Mental Hygiene, Bureau of STI, New York City, USA

10.1136/sextrans-2019-sti.272

Background Expedited partner therapy (EPT) is not recommended for U.S. men who have sex with men (MSM), due, in part, to concerns that STI and HIV infections may remain undiagnosed in EPT-treated partners who do not seek medical attention. To estimate how often infections might be missed, we assessed bacterial STI and new HIV diagnoses among MSM presenting as contacts to Chlamydia trachomatis (Ct) or Neisseria gonorrhoeae (GC).

Methods MSM attending New York City sexual health clinics are routinely tested for HIV, syphilis, and urogenital and extra-genital $\mathrm{Ct}$ and GC. We measured the number and percent of visits, during 2016-2018, with diagnoses of new HIV infection, or alternate/additional bacterial STI, among MSM who had: reported contact to STI, a clinician diagnosis of contact to GC or CT, and no reported contact to syphilis.

Results A total of 3,549 MSM had 4,390 visits eligible for analysis. Overall, 14.6\% (640/4,390) visits resulted in bacterial STI diagnoses other than those to which exposure was reported. Among MSM-visits for exposure to Ct-only, $12.4 \%$ $(177 / 1,430)$ resulted in GC diagnoses (including 81 rectal GC infections). Syphilis was diagnosed at $4.4 \%(159 / 3,652)$ of visits for $\mathrm{Ct}$ or GC exposure (49 primary/secondary, 49 early latent, 61 late latent). Twenty-eight new HIV diagnoses were made (3 acute, 25 non-acute infections); 8 among visits for Ct-only exposure, 20 for GC exposure.
Conclusion MSM reporting contact to Ct or GC, have other, concurrent bacterial STI that will be inadequately treated with therapy directed at only the STI to which they report exposure. A substantial number of HIV infections may remain undiagnosed if sex partners to MSM with Ct and GC do not receive HIV testing. Opportunities to offer HIV pre-exposure prophylaxis may also be missed. Our findings support examining and testing MSM exposed to Ct or GC rather than using EPT.

Disclosure No significant relationships.

\section{P076 DO PRESCRIPTIONS FOR EXPEDITED PARTNER THERAPY GET FILLED? FINDINGS FROM A MULTI-JURISDICTIONAL EVALUATION, US, 2017-2018}

${ }^{1} J u l i a$ Schillinger*, ${ }^{2}$ Jennifer Slutsker, ${ }^{2}$ Lai-Yi Tsang, ${ }^{1}$ Susan Blank. ${ }^{1}$ Centers for Disease Control and Prevention, Division of STD Prevention, New York City, USA; ${ }^{2}$ New York City Department of Health and Mental Hygiene, Bureau of STI, New York City, USA

\subsection{6/sextrans-2019-sti.273}

Background Expedited partner therapy (EPT) is commonly provided by prescription, however, the effectiveness of prescription-EPT has not been studied, and will depend on how often prescriptions are filled. We examined whether EPT-prescriptions get filled at retail pharmacies when the cost barrier is removed. Methods Clinical sites diagnosing large numbers of Chlamydia trachomatis $(\mathrm{Ct})$ infections and providing EPT-prescriptions were recruited. An industry partner developed voucher cards redeemable, with prescription, for free azithromycin, $1 \mathrm{~g}$, in any pharmacy in states where EPT is legal. Voucher cards were distributed to clinical sites. Providers prescribing EPT recorded index-patient age, gender, and prescription date on a tear-off tab accompanying each card, retained these tabs, and dispensed vouchers, along with EPT-prescriptions, to index-patients. A standard pharmacy interface captured unique voucher codes, prescription data, age, sex of the person redeeming the card. Pharmacy data were downloaded from an industry portal, linked with data from tear-off tabs, and analyzed. We considered redeemed cards a surrogate for filled prescriptions, and assessed patient and provider characteristics associated with redemption.

Results During September, 2017-June, 2018, 30 sites in New York City (NYC), New York State outside NYC, and Maryland enrolled; 580 EPT discount cards were dispensed by providers and 40\% (234/580) redeemed at pharmacies. Redemption differed significantly by: index-patient gender (women, 44\% versus men, 32\%, $\mathrm{p}=0.005)$, and age in years $(\leq 18,23 \%$ versus $>18,45 \%, \mathrm{p}<0.001)$. Most cards $(57 \%)$ were redeemed sameday. Among sites dispensing $\geq 10$ cards, redemption was $41 \%$ (212/521), range 6-78\% (median, 36\%); redemption of cards distributed at STD clinics was 38\% (51/136). After excluding a high-volume site with an onsite pharmacy, there was no association between index-patient gender and card redemption.

Conclusion Less than half of EPT-prescriptions were filled, despite medication being dispensed for free, suggesting prescription-EPT to treat $\mathrm{Ct}$ may result in low partner-treatment rates, especially among adolescents.

Disclosure No significant relationships. 